\title{
Change in nostril ratio after cleft rhinoplasty: correction of nostril stenosis with full-thickness skin graft
}

Joong Min Suh, Ki Il Uhm

Department of Plastic and Reconstructive Surgery, Kangdong Sacred Heart Hospital, Seoul, Korea
Background: Patients with secondary deformities associated with unilateral cleft lip and nose might also suffer from nostril stenosis due to a lack of tissue volume in the nostril on the cleft side. Here, we used full-thickness skin grafts (FTSGs) to reduce nostril stenosis and various methods for skin volume augmentation. We compared the changes in the symmetry of both nostrils before and after surgery.

Methods: From February 2016 to January 2020, 34 patients underwent secondary cheiloplasty and open rhinoplasty for secondary deformities of the unilateral cleft lip and nose with nostril stenosis. FTSG was used on the nostril floor, nasal columella, and alar inner lining. The measured nasal profile included the nostril surface, nostril circumference, width of the nostril floor, and distance from the alar-facial groove to the nasal tip. The "overlap area," which was defined as the largest overlapping area when the image of the cleft nostril was flipped to the left and right and overlaid on the image of the normal side nostril, was also calculated. The degree of symmetry was evaluated by dividing the value of the cleft side by that of the normal side of each measured profile and expressed as "ratios."

Results: The results of all profile ratios, except for the nostril floor width, became significantly close to 1 , which represents full symmetry. The overlap area ratio improved from $62.7 \%$ to $77.3 \%$, meaning that the length and width of the nostril as well as the overall shape became similar $(p<0.05)$.

Conclusion: When performing cleft rhinoplasty with nostril stenosis, FTSG is useful to achieve symmetry in the nostril size and shape. Skin grafting is simpler to perform than the other types of local flap, and the results are generally satisfactory.

Keywords: Cleft lip / Humans / Nose / Pathologic constriction / Rhinoplasty

\section{INTRODUCTION}

Unilateral nostril stenosis is a condition in which the shape and

\author{
Correspondence: Ki Il Uhm \\ Department of Plastic and Reconstructive Surgery, Kangdong Sacred Heart \\ Hospital, 150 Seongan-ro, Gangdong-gu, Seoul 05355, Korea \\ E-mail: kiumps@hanmail.net \\ This article was presented at the 10th Research and Reconstruction Forum on July \\ 24, 2020, in Korea. \\ Received January 28, 2021 / Revised March 22, 2021 / Accepted April 19, 2021
}

size of one nostril are smaller than the those of the other nostril. Unilateral nostril stenosis can be congenital in patients with unilateral cleft lip nose deformity or might be caused by trauma, infection, or iatrogenic causes such as repetitive open rhinoplasty or weir surgery. Several methods can be used to treat nostril stenosis, including surgery using the V-Y advancement flap, para-alar pedicle flap and alar transposition flap, Z-plasty, and alar web excision [1-5]. However, although these methods can improve the shape of both nostrils in the short term, be- 
cause the nostril on the side with stenosis has lesser skin volume than that on the normal side, there is a high probability of recurrence of the stenosis due to postoperative scar contracture.

The goal of surgery in patients with secondary deformities of the unilateral cleft lip and nose is to restore the symmetry of the overall shape of the nose. To achieve this goal, we symmetrized the shape of both nostrils and performed tip projection in cases where it was possible; thus, the surgery was performed to improve the overall esthetics of the shape of the nose. We widened the stenotic nostrils using full-thickness skin grafts (FTSGs) harvested from the postauricular area and augmented the skin volume using various methods, including B-flap and C-flap interdigitation, orbicularis oris muscle approximation, septal cinching suture, and autogenous or allogeneic bone grafting for secondary deformities of the unilateral cleft lip and nose with nostril stenosis. The outcomes were assessed for changes in the symmetry of both nostrils before and after surgery.

\section{METHODS}

\section{Patients}

This study was approved by the appropriate institutional review board and performed in accordance with the principles of the Declaration of Helsinki. The requirement of obtaining informed consent was waived because of the retrospective nature of the study, and the analysis was performed using anonymous clinical data. From February 2016 to January 2020, data of patients who underwent secondary cheiloplasty and open rhinoplasty were reviewed retrospectively. Patients underwent cheiloplasty and secondary cleft rhinoplasty, and FTSG was performed on the nostril floor, columella, and alar inner lining according to the location and shape of the inadequate skin volume causing nostril stenosis. In patients with a narrow nostril

Table 1. Patient demographics and sites of FTSG

\begin{tabular}{lc}
\hline Variable & No. (\%) \\
\hline No. of patients & \\
Male & $24(70.6)$ \\
Female & $10(29.4)$ \\
Age, yr & \\
Mean \pm SD & $26.2 \pm 12.6$ \\
Range & $15-65$ \\
Sites of FTSG performed & \\
Columella & $14(41.2)$ \\
Nostril base & $23(67.6)$ \\
Alar inner lining & $9(26.5)$ \\
Total & 34 \\
\hline
\end{tabular}

FTSG, full-thickness skin graft. floor, FTSG was used to widen the nostril floor. In case of inadequate columella growth or short columella due to scars from previous open rhinoplasty, a triangular FTSG was placed on the outer lining of the columella. In cases with nostril constriction due to lack of skin on the nostril inner lining, FTSG was used after releasing the constriction. The FTSG was harvested from the postauricular area, and the graft included the skin, dermis, and subcutaneous fat, similar to the thickness of the surrounding tissue.

All surgeries were performed by a senior plastic surgeon (KIU). The study subjects comprised patients with nostril stenosis in whom the size of the nostril on the cleft side was smaller than that of the other nostril due to severe secondary deformities of the unilateral cleft lip and nose. Patients in whom the area of the nostril on the cleft side was larger or equal to that of the nostril on the normal side were excluded. Thirty-four patients met the inclusion criteria ( 24 males and 10 females). The mean patient age was 26.2 years (range, $15-65$ years), and the average follow-up period was 8.4 months (Table 1).

A frontal picture and worm's eye view of all patients were captured with the same setting for all follow-ups before and after surgery. The area of the nostril on the normal and cleft sides, the width of the nostril floor, and the distance from the alar-facial groove to the nasal tip were measured (Fig. 1). The "overlap area" was also calculated, which was defined as the largest overlapped area when the image of the cleft nostril flipped to the left and right and overlied to the image of the normal side nostril (Fig. 2). All nasal profiles were measured manually using Adobe Photoshop and CS6 digital pixel values to accurately calculate the curve profiles.

Since pictures of the same patient can have slightly different

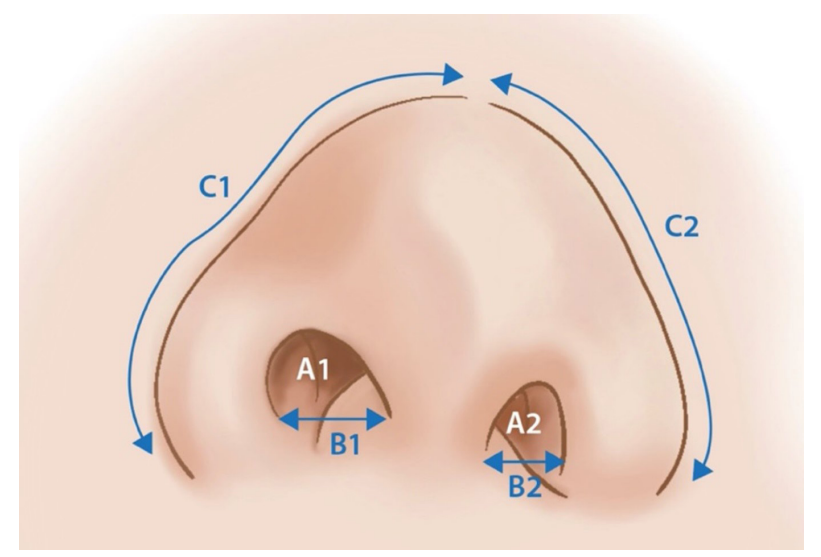

Fig. 1. A schematic diagram of nasal profiles. A1, right nostril surface; A2, left nostril surface; B1, right nostril floor width; B2, left nostril floor width; $\mathrm{C} 1$, right side distance from the alar-facial groove to the nasal tip; C2, left side distance from the alar-facial groove to the nasal tip. 
perspectives and angles, it is not appropriate to quantitatively compare the measured pixel values. The degree of symmetry was evaluated by dividing the value of the cleft side by the that of the normal side of each measured profile, and expressed in "ratios." Therefore, a ratio of 1 represents full symmetry. The ratios were thus used to quantify the symmetry and to compare

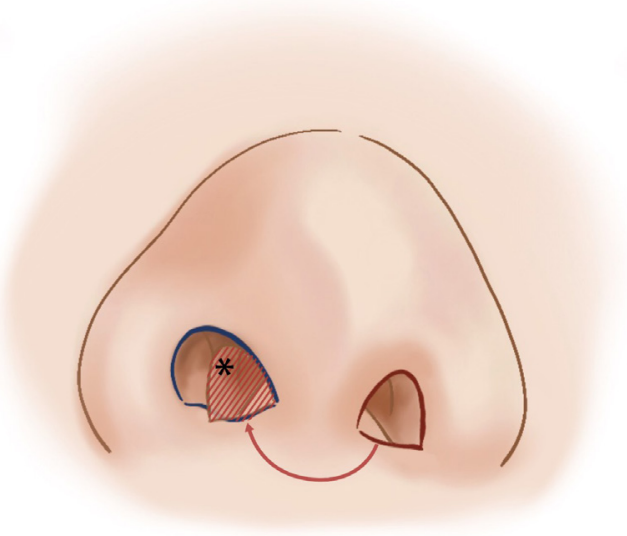

Fig. 2. The "overlap area" was defined as the largest overlapped area when the image of the cleft nostril flipped to the left and right and overlied to the image of the normal side nostril. The overlap area ratio was calculated by dividing the normal nostril area by the hatched asterisk $\left(^{*}\right)$ area. the changes in symmetry before and after surgery.

Statistical analysis was performed using IBM SPSS version 23 (IBM Corp., Armonk, NY, USA). The nasal profiles calculated before and after surgery in each patient were analyzed using a paired $t$-test. $p$-values $<0.05$ were considered statistically significant.

\section{Surgical technique}

All surgeries were performed under general anesthesia. Secondary cheiloplasty was performed for deformity of the upper lip and philtrum and was followed by secondary cleft rhinoplasty. In addition, nostril floor depression on the cleft side and straightening of the slanted columella were performed before open rhinoplasty. Subsequently, the nostril stenosis was corrected using FTSG in earnest, and the overall shape of the nose was improved through alar cartilage rearrangement.

To elevate the nostril floor, the vertical length of the nostril floor was first increased by B-flap and C-flap interdigitation, and the horizontal width of the nostril floor was widened by grafting a half-moon-shaped FTSG on the nostril floor (Fig. $3 \mathrm{~A})$. If the horizontal length of the nostril was adequate, orbicularis oris muscle approximation was performed through an intraoral approach to elevate the depressed nostril floor on the
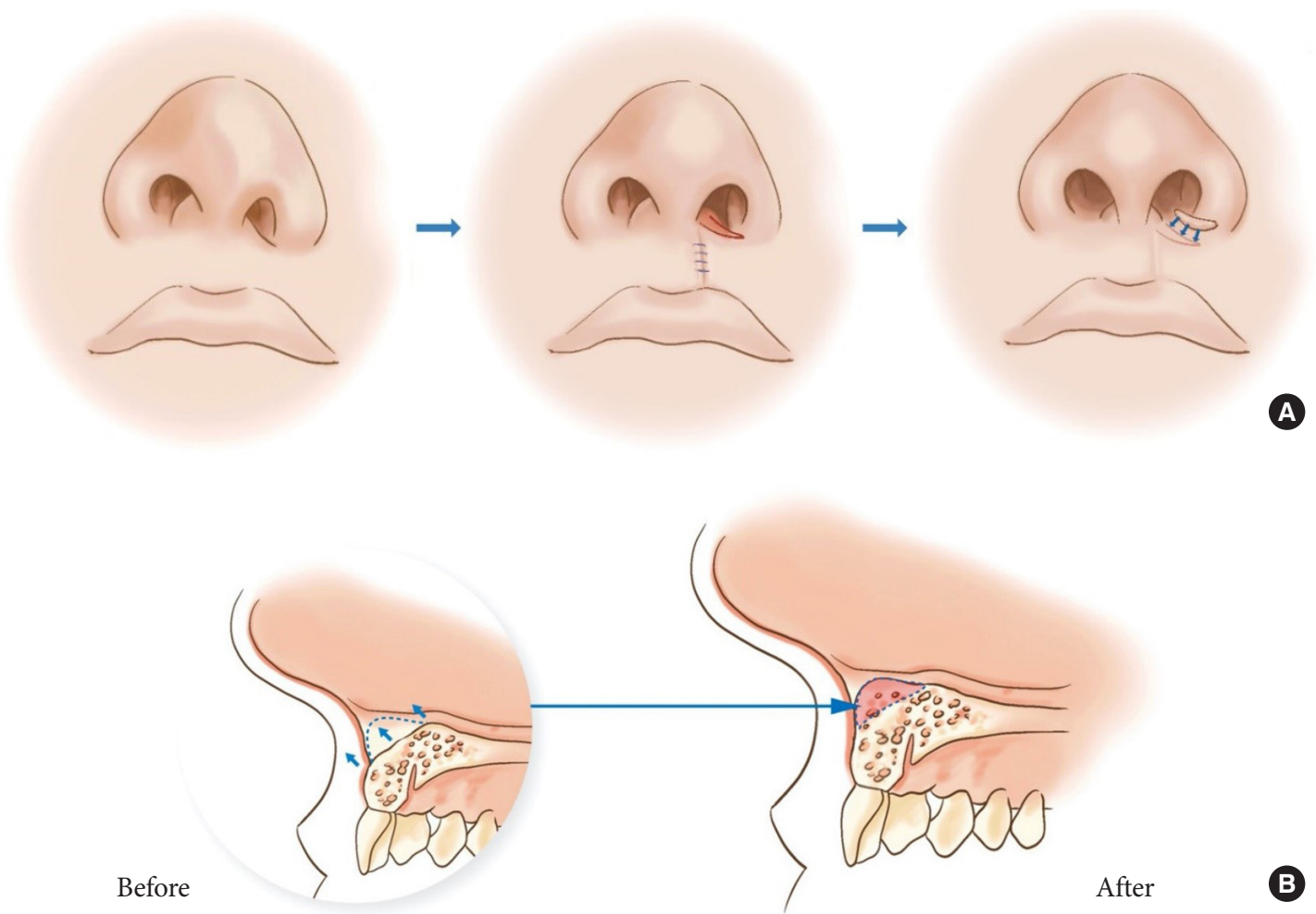

Fig. 3. (A) B-flap and C-flap interdigitation and full-thickness skin graft can lengthen skin deficiency in the nostril floor. (B) Schematic sagittal view of a depressed nostril floor. The nostril floor can be elevated with an autologous or allogeneic bone graft; this can be achieved by performing an autologous dermal graft, orbicularis oris muscle approximation, and septal suspension suture. 

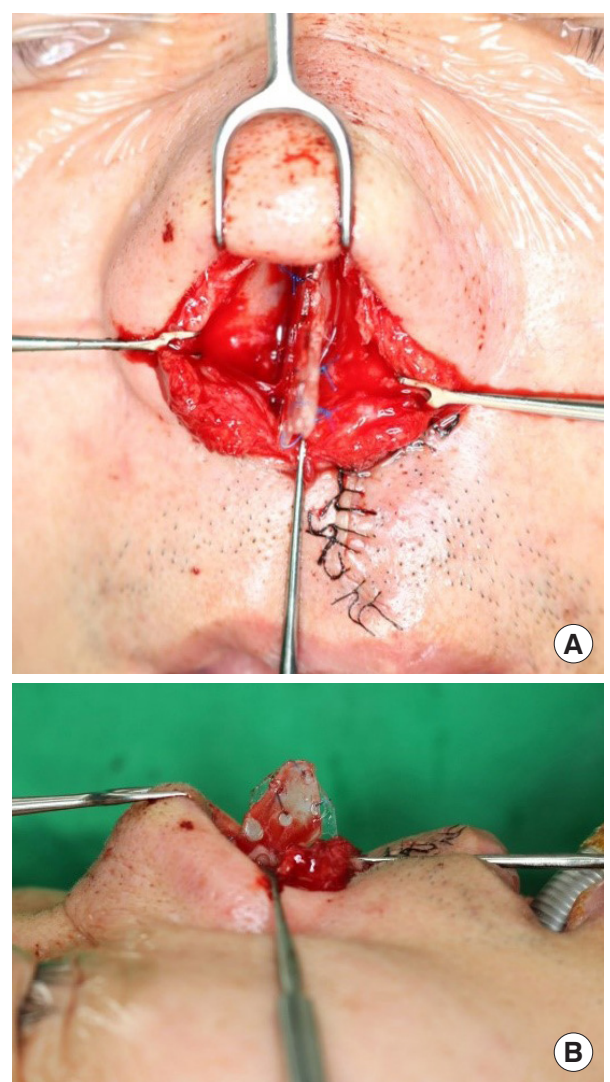

Fig. 4. Septal extension graft performed with absorbable mesh. The harvested septal cartilage was sutured at the tip of the mesh. (A) Anteroposterior view. (B) Lateral view.

cleft side, and a septal cinching suture was performed to erect the slanted footplate. In addition, autogenous or allogeneic bone grafting was performed on the base of the pyriform aperture to elevate the cleft nostril floor (Fig. 3B).

In open rhinoplasty, alar cartilage rearrangement was performed following a septal extension graft using an absorbable mesh, and nostril stenosis was corrected by using FTSG to cover the absence of skin at the incision site of the columella and the inner alar lining. The nasal cutaneous flap was elevated through a marginal and transcolumellar incision. Submucosal resection was performed by approaching the subperichondrium plane using a Metzenbaum scissor and Joseph periosteal elevator to expose the septal cartilage, which was harvested for the septal extension graft. The absorbable mesh was then inserted using the anterior nasal spine and the tip of the nasal bone as the base and fixed to serve as a support for the tip projection (Fig. 4). Additionally, the harvested septal cartilage was sutured in a batten-type using 5-0 Prolene (Ethicon, Inc., Somerville, NJ, USA) at the end of the mesh. To prevent necrosis of the nose tip, tip plasty was performed on the protruding part of the mesh with an onlay graft with autologous cartilage

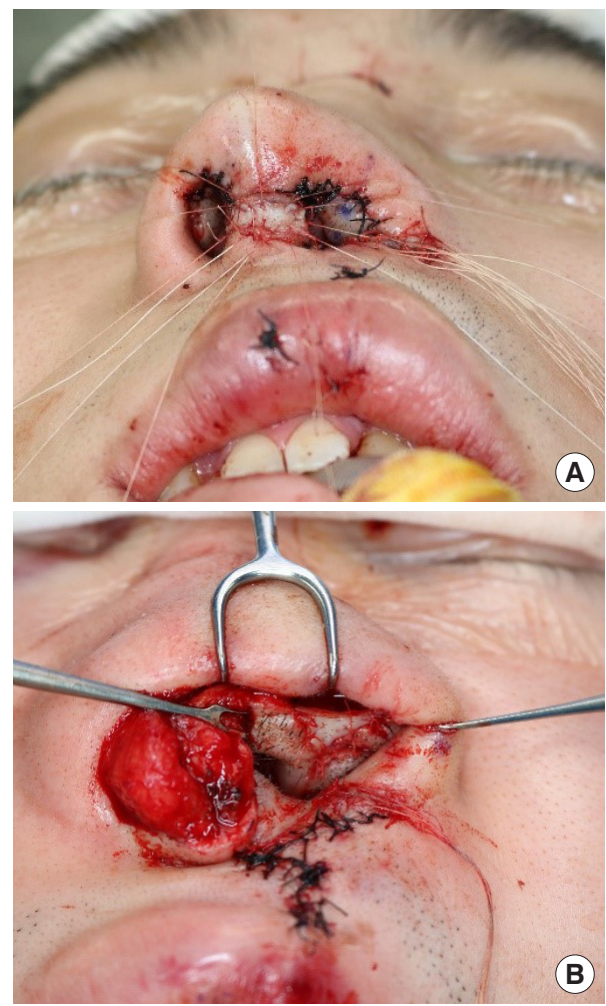

Fig. 5. A skin graft is required to increase the skin deficit while closing the open rhinoplasty incision. (A) Full-thickness skin graft performed on the columella. (B) As the nostril circumference is lengthened, a skin graft was also required for the alar inner lining; this was performed at the end of the marginal incision on the left nostril.

or dermis. Triangular FTSG was applied to the columella to increase the nostril circumference and facilitate tip projection (Fig. 5A). A cut back incision was made at the end of the marginal incision on the cleft side, and a triangular FTSG was used to extend the length of the inner alar lining (Fig. 5B). Subsequently, all FTSG sites were dressed with a tie-over dressing so that skin grafts could be taken without complications such as hematoma. The tie-over dressings were removed 7 days after surgery, and the stitches were removed according to the degree of improvement at the surgical site. Following healing of all the surgical sites, a silicone stent (Koken Co., Ltd., Tokyo, Japan) was placed during the wound remodeling period to manipulate the nostril into a symmetrical shape.

\section{RESULTS}

The nasal profile measurements were obtained from the preoperative and postoperative evaluation of the patients, and the profile ratios were all closer to 1 after surgery (Table 2). Postoperative symmetry was significantly improved in the nostril area measured from the front, the nostril area measured in the 
Table 2. Variations of nasal profiles after surgery

\begin{tabular}{|c|c|c|c|c|}
\hline Variable & $\begin{array}{l}\text { Preoperative } \\
\text { ratio }(\%)\end{array}$ & $\begin{array}{l}\text { Postoperative } \\
\text { ratio (\%) }\end{array}$ & $\begin{array}{l}\text { Change } \\
(\%)\end{array}$ & $p$-value \\
\hline Nostril surface ${ }^{a)}$ & & & & $<0.05$ \\
\hline Worm's eye view & 70.3 & 87.0 & 16.6 & \\
\hline Anteroposterior view & 57.7 & 82.3 & 24.7 & \\
\hline Nostril circumference ${ }^{\text {b) }}$ & 82.6 & 92.7 & 10.1 & $<0.05$ \\
\hline Nostril floor widtha) & 93.4 & 99.7 & 6.3 & 0.062 \\
\hline $\begin{array}{l}\text { Alar-facial groove to tip } \\
{\text { distance })^{()}}^{\text {a }}\end{array}$ & 82.9 & 92.3 & 9.5 & $<0.05$ \\
\hline Overlap area) & 62.7 & 77.3 & 14.6 & $<0.05$ \\
\hline
\end{tabular}

Values were measured and calculated based on the schematic diagram in Fig. 1. $A 1$, right nostril surface; $A 2$, left nostril surface; $B 1$, right nostril floor width; B2, left nostril floor width; $\mathrm{C} 1$, right side distance from the alar-facial groove to the nasal tip; $\mathrm{C} 2$, left side distance from the alar-facial groove to the nasal tip.

${ }^{\text {a) }} \mathrm{A} 2$ area/A1 area; ${ }^{\text {b)}} \mathrm{A} 2$ circumference/A1 circumference; ${ }^{\text {clB }}$ 2 length/B1 length; ${ }^{\text {d) }} \mathrm{C} 2$

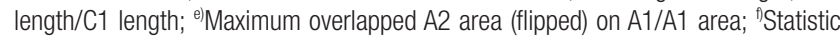
analysis with paired $t$-test.

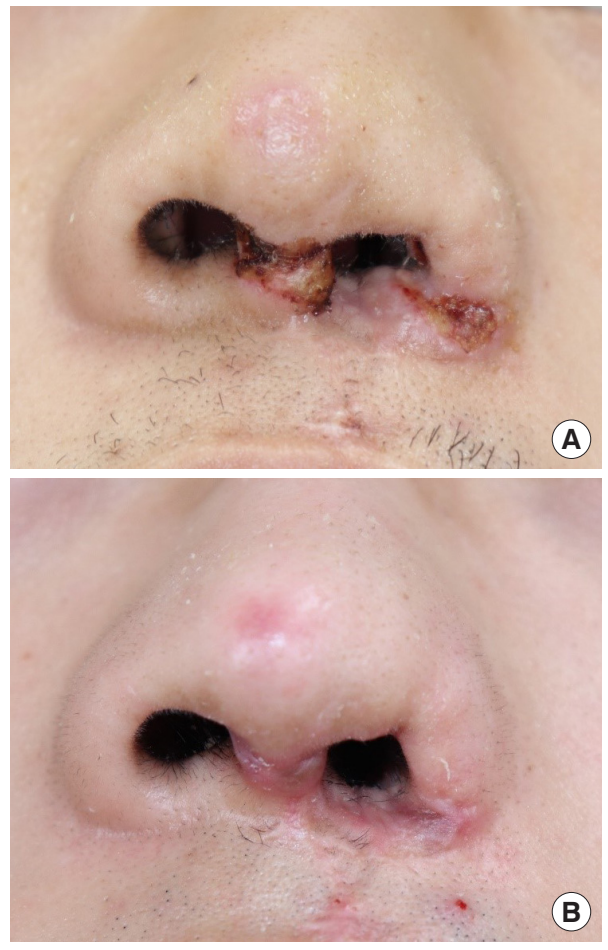

Fig. 6. (A) A 20-year-old male underwent full-thickness graft on the columella and left nostril floor. The postoperative view at 12 days shows partial graft necrosis on the columella in the graft on the nostril floor. (B) Improvement with secondary healing at postoperative 30 days.

worm's eye view, the circumference of the nostril, and the distance from the alar-facial groove to the nasal tip. The nostril floor width ratio demonstrated $6.3 \%$ improvement in symmetry; however, the difference was not statistically significant $(p=0.062)$. The overlap area ratio was improved from $62.7 \%$ to $77.3 \%$, which means that not only the length and width of the nostril, but the overall shape has become similar $(p<0.05)$.
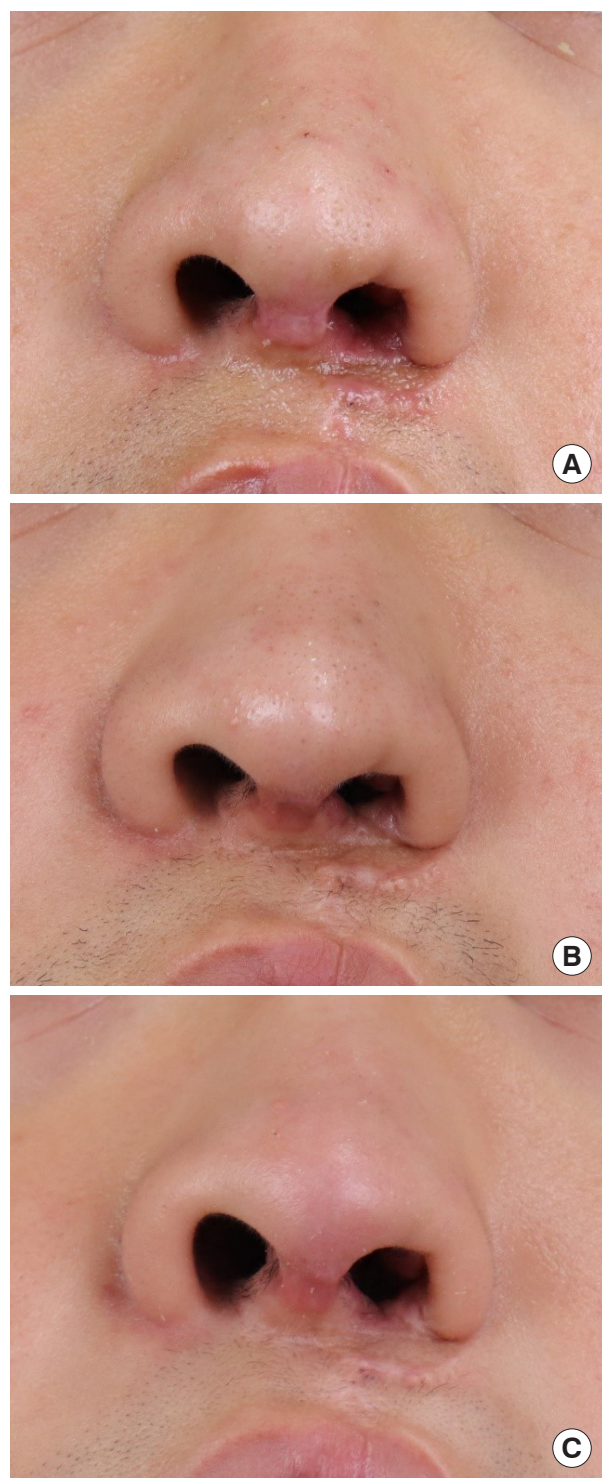

Fig. 7. A full-thickness skin graft is aesthetically pleasing following scar maturation. An 18-year-old male patient underwent full-thickness skin graft on the nostril floor and columella. (A) One month after surgery, the skin graft on the columella shows erythematous scarring, and that of the nostril floor shows erythematous and elevated scarring. (B) Four months after surgery, elevated and erythematous scarring was alleviated and also appears to be pigmented. (C) Seven months after surgery, the wound remodeling period has passed and the graft site possesses a natural texture, similar to that of the surrounding tissue.

In some patients, complications such as partial necrosis occurred during skin graft healing, all of which resolved with secondary healing without the need for surgical intervention (Fig. 6). The skin grafts were observed as red, elevated, pigmented scars at the time of remodeling and became similar to the surrounding tissues in terms of color and texture over time (Fig. 7). Representative photographs of the results are shown in Figs. 8 and 9. 

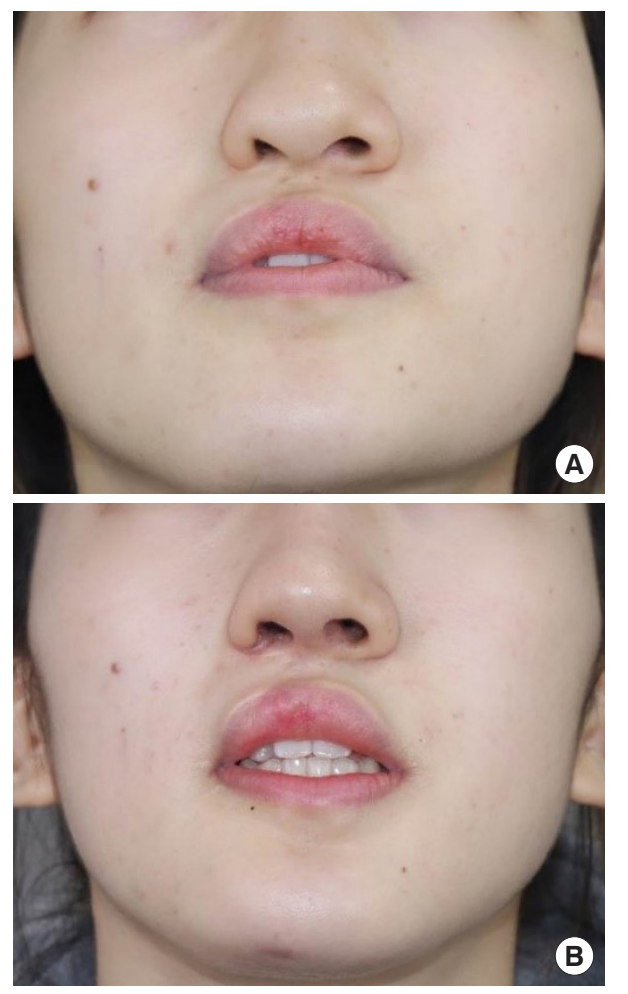

Fig. 8. (A) A 20-year-old female patient had a stenosis on the cleft side of her nose. She underwent secondary rhinoplasty and received full-thickness skin grafts on her nostril floor. (B) Photographs taken 9 months after the surgery demonstrating enlarged nostril circumferences.

\section{DISCUSSION}

The characteristics of secondary deformities of the cleft lip and nose become more pronounced with the onset of puberty. The nasal dorsum deviates to the normal side, and the nostril floor on the cleft side shows a depression. As the nasal septum deviates to the normal side, the columella slants toward the cleft side, and the footplate on the cleft side weakens. The cleft-side ala flares and droops down, and the shape of the nostril becomes horizontal due to the widening of the alar base [3]. These changes occur due to abnormal alignment of the orbicularis oris muscle, and deformations occur due to hypoplasia of the skin and soft tissue around the pyriform aperture. In patients with severe cleft nose deformity, nostril stenosis occurs when the tissue volume around the nostril on the cleft side is inadequate, and surgery to correct this is challenging.

Several surgical methods have been attempted to correct this deformity. Straith devised Z-plasty and Millard devised a forked flap to extend the length of the columella $[1,2]$. Blandini et al. [4] proposed lengthening the nostril inner lining with a vestibular labial mucosal flap. In addition, transposition flaps in the

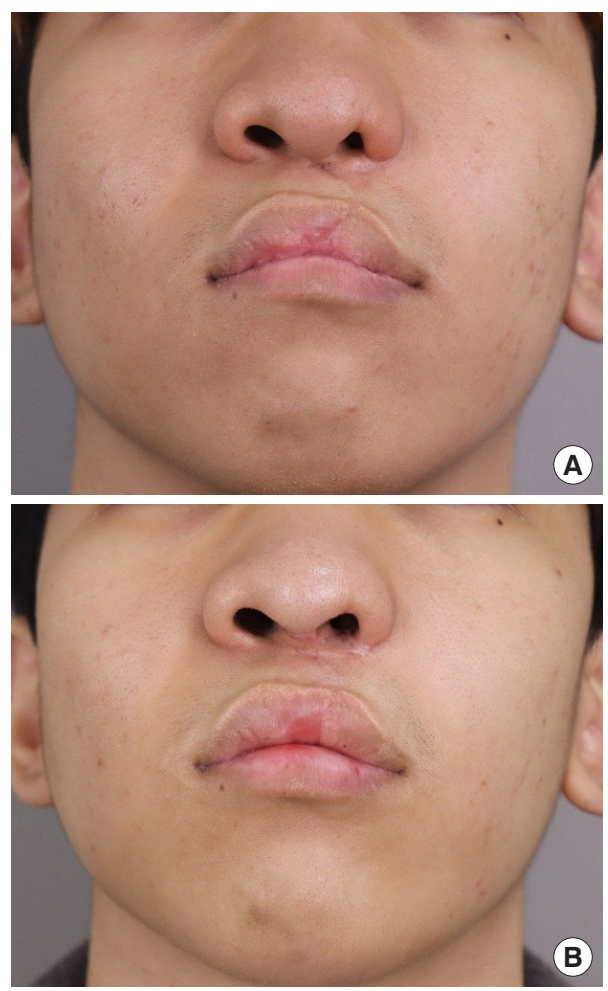

Fig. 9. (A) A 20-year-old male patient had a severe stenosis on the cleft side of nose. He underwent a septal extension graft with absorbable mesh and received full-thickness skin grafts on his columella, nostril floor, and alar inner lining. (B) Photographs taken 12 months after the surgery demonstrating satisfactory improvement in both nostril size and shape.

peri-alar nasolabium can increase the skin of the nostril sill [5]. However, because the current methods involve manipulating the existing or connected tissues, they are unsuitable when the degree of nostril stenosis is severe and do not resolve the defects of the skin and soft tissues. About 40 years ago, Rees et al. [6] used an FTSG to correct the nostril vestibule fold. Further, Cheon and Park [7] and Lee et al. [8] showed good results in lengthening the short columella in patients with bilateral cleft lip and nose deformity using skin-subcutaneous composite grafts and full-thickness grafts, respectively.

In this study, we used two techniques to correct the lip deformity associated with nostril stenosis. The first was to extend the length around the nostril, and the second was to symmetrize the shape of both nostrils. The nostril on the cleft side has a shorter circumference than that on the normal side. Therefore, while performing a septal extension graft in cleft rhinoplasty, FTSG harvested from the posterior auricular area was applied to extend the length of the area where necessary.

In addition, as the septum is deviated from the midline to the normal side, the columella is slanted, and the normal footplate protrudes more than that on the cleft side. We corrected this 

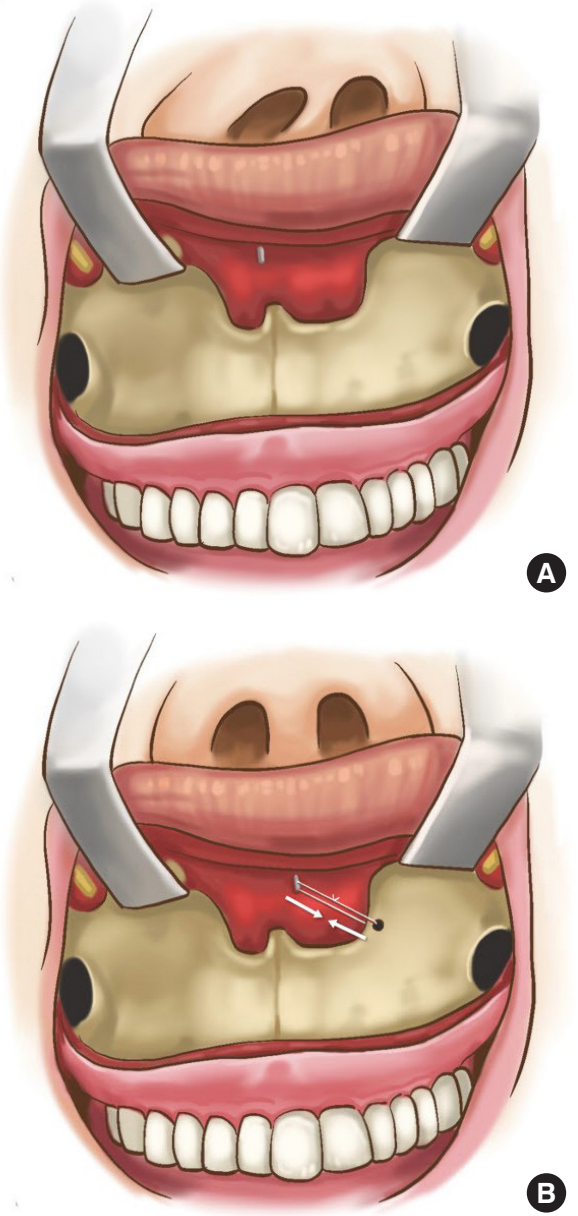

Fig. 10. (A) The caudal end of the deviated septum should be detached from the anterior nasal spine during dissection. (B) Septal cinching suture involves suturing the caudal end of the septum and pulling it to the cleft side of the pyriform aperture base; this functions to straighten the slanted columella and corrects the footplate protruding toward the normal nostril.

discrepancy by performing a septal cinching suture that pulls and fixes the deviated septum to the pyriform aperture base on the cleft side (Fig. 10). However, as the septal cinching suture performed at this time makes the normal nostril larger and the nostril on the cleft side smaller, it is necessary to secure an adequate horizontal length by performing grafting using FTSG on the cleft nostril floor before septal cinching. In addition, to compare the shape of the nostril on both sides, we flipped the image of the nostril at the cleft, and overlapped it to compare both sides in terms of the extension of the nostril circumference. We evaluated the improvement in shape of both nostrils after surgery, confirming the successful achievement of our two goals (Table 2).

In patients with nostril stenosis, the nostril floor is depressed, similar to that in other patients; hence, it should be corrected at the same time. The orbicularis oris muscle approximation to el- evate the nostril floor cannot be performed because the horizontal length of the nostril is shortened, and the dermal graft under the FTSG to extend the length cannot be used because it interferes with the blood supply of the graft. Therefore, in this case, the nostril floor should be elevated by performing autogenous or allogeneic bone grafting on the base of the pyriform aperture. Alternatively, grafting using FTSG may be performed prior to surgery to correct the depression after the graft is completely taken.

Complications such as loss of skin graft, scar deformities, and re-stenosis can occur at the FTSG sites. Moreover, as skin grafts are performed in areas with poor blood supply, such as scar tissue or on the columella from previous surgery, it is necessary to prepare a healthy bed with careful dissection during surgery. In addition, after surgery, tie-over dressings should be maintained for an adequate period of time to allow compression between the bed and allow the graft to adhere well.

This study has several limitations. First, since each parameter was measured manually by one person, there might be a measurer's bias. Second, unlike the normal nostril, the shape of the nostril on the cleft side is irregular and the anatomical landmark is ambiguous, which might cause an error in the measured value. The results might be more reliable if measurements are taken by multiple people.

FTSG is effective even in patients with severe nostril stenosis because it can provide a relatively large skin volume with little donor site morbidity. In addition, it is relatively easy to perform, and long-term follow-up has shown that complications such as re-stenosis are rare. It is an esthetically pleasing procedure that results in good color matching with the surrounding tissues.

\section{NOTES}

\section{Conflict of interest}

No potential conflict of interest relevant to this article was reported.

\section{Ethical approval}

The study was approved by the Institutional Review Board of Kangdong Sacred Heart Hospital (IRB No. 2021-01-007) and performed in accordance with the principles of the Declaration of Helsinki. The requirement of obtaining informed consent was waived because of the retrospective nature of the study.

\section{Patient consent}

The patients provided written informed consent for the publication and the use of their images. 


\section{ORCID}

Joong Min Suh https://orcid.org/0000-0002-3475-091

Ki Il Uhm https://orcid.org/0000-0002-5343-9899

\section{Author contribution}

Conceptualization: KIU. Data curation, formal analysis: JMS. Methodology: KIU. Project administration: JMS. Visualization, software: JMS. Supervision, validation: KI Uhm. Writing - original draft: JMS. Writing - review \& editing: JMS.

\section{REFERENCES}

1. Millard DR. Bilateral cleft lip and a primary forked flap: a preliminary report. Plast Reconstr Surg 1967;39:59-65.

2. Straith CL. Elongation of the nasal columella; a new operative technique. Plast Reconstr Surg (1946) 1946;1:79-86.

3. Sadove R, Ladaga L, Magee WP Jr. Cartilaginous histology of the cleft lip nose: proving the extrinsic etiology. Plast Reconstr
Surg 1988;81:655-61.

4. Blandini D, Tremolada C, Beretta M, Mascetti M. Iatrogenic nostril stenosis: aesthetic correction using a vestibular labial mucosa flap. Plast Reconstr Surg 1995;95:569-71.

5. Costin A, Antonio AM, Goulao J. Perialar semilunar advancement flap for the reconstruction of nasal sidewall defects: our experience. J Cutan Aesthet Surg 2019;12:71-3.

6. Rees TD, Guy CL, Converse JM. Repair of the cleft lip-nose: addendum to the synchronous technique with full-thickness skin grafting of the nasal vestibule. Plast Reconstr Surg 1966; 37:47-50.

7. Cheon YW, Park BY. Long-term evaluation of elongating columella using conchal composite graft in bilateral secondary cleft lip and nose deformity. Plast Reconstr Surg 2010;126:543-53.

8. Lee YS, Shin DH, Choi HG, Kim JN, Lee MC, Kim SH, et al. Columella lengthening with a full-thickness skin graft for secondary bilateral cleft lip and nose repair. Arch Plast Surg 2015; 42:704-8. 